\title{
REDUCED-DYNAMIC PRECISE ORBIT DETERMINATION FOR LOW EARTH ORBITERS BASED ON HELMERT TRANSFORMATION
}

\author{
Junping Chen \\ GeoForschungsZentrum Potsdam,Telegrafenberg A17,14473 Potsdam, Germany \\ junping.chen@gfz-potsdam.de \\ Jiexian Wang \\ Department of Surveying and Geo-informatics,Tongji University,200092 Shanghai, China. \\ wangjiexian@mail.tongji.edu.cn
}

\begin{abstract}
A model based on Helmert transformation is presented in reduced-dynamic Precise Orbit Determination(POD). As an implementation, a reduced-dynamic POD approach was developed. The approach includes two steps: firstly, kinematic POD and then reduced-dynamic POD. Based on the approach, a set of programs were developed. POD of CHAMP and GRACE was then carried out. Kinematic and reduced-dynamic POD for CHAMP and GRACE satellite over 2 weeks time show that reduced-dynamic orbits of CHAMP have a mean 3D RMS of $0.26 \mathrm{~m}$ compared to PSO orbit of GFZ, and the mean 3D RMS of GRACE-A has the same value compared to GNV1B orbit of JPL. The 3D RMS is reduced by up to $40 \%$ compared to kinematic solutions.
\end{abstract}

Keywords: Reduced-dynamic precise Orbit Determination, Helmert transformation, GPS, LEO

\section{INTRODUCTION}

In last years, several gravity satellite missions like CHAMP(CHAllenging Minisatellite Payload, http://www.gfz-potsdam.de/pb1/op/champ/index_CHAMP.html) and GRACE(Gravity Recovery And Climate Experiment, http://www.gfz-potsdam.de/pb1/op/grace/index_GRACE.html) were launched, a lot of scientific work of precise orbit determination has been carried out since then (Reigber et al(eds.),2003). To summarize, the POD method can be divided into dynamic method and kinematic method according to the theory and observations it used (D. Svehla, 2003; S. Zhu et al, 2004; Chen, 2007). In the dynamic method, the orbit precision mainly depends on initial orbital elements and dynamic models in satellites' motion equation. Benefiting from efforts of the International Earth Rotation and Reference Service (IERS, http://www.iers.org/) and other communities, dynamic models improve significantly in recent years. In the kinematic method, main challenge is how to reduce influence of weak geometry and phase breaks. One of the kinematic POD methods developed by Beutler and Bock (G. Beutler, 2004; H. Bock, 2003) implements the combination of kinematic POD and reduced-dynamic POD. In their method, 
the reduced-dynamic orbits are used as a priori orbits for the data pre-screening in kinematic POD, and the resulting kinematic orbits are used as pseudo-observations for reduced-dynamic POD. This procedure runs iteratively, the best orbits that can be achieved are the reduced-dynamic orbits (H. Bock, 2003).

The model in their reduced-dynamic POD method is basically the same as what is used in the orbit fitting for GPS (G. Beutler, 1995), i.e., the kinematic orbits are treated as "true" orbits (pseudo-observations). As a consequence, the errors of kinematic orbits will directly go into the reduced-dynamic orbits. The GPS orbits used in their kinematic POD are from IGS final combination, therefore the corresponding reference system of their kinematic orbits is consistent with the frame defined by the IGS final solution. But as we know that each POD software may differ in dynamic models and processing approaches, consequently the software difference results in the difference of the reference frame defined. Therefore directly treating kinematic orbits as pseudo-observations in reduced-dynamic POD may distort the reference frames.

To solve these problems, we suggest a reduced-dynamic model, which connects the dynamically integrated orbits and the kinematic orbits using Helmert transformation. Using this model, the errors of kinematic orbits can be partly absorbed and therefore the dependence of reduced-dynamic POD on kinematic orbits can be reduced. On the other hand, the system difference between kinematic orbits and reduced-dynamic orbits are properly modeled, in which the model is the same as what is used in the IGS final orbit combination (G. Beutler, 1995). Based on this model, reduced-dynamic POD of CHAMP and GRACE satellites was carried out. Our results show that 3D RMS of residuals (compared with reference orbits) can be reduced up to $40 \%$ compared to kinematic solutions.

\section{ORBIT INTEGRATION}

According to dynamic POD theory, the satellites' motion equation and satellites' initial orbits at epoch $t_{0}$ can be written as,

$$
\left\{\begin{array}{l}
\dot{x}=F(x, t) \\
\left.x\right|_{t_{0}}=x_{0}
\end{array}\right.
$$

Where, $x_{0}=\left(\begin{array}{lll}r_{0} & \dot{r}_{0} & p_{0}\end{array}\right)^{T}$ are initial orbits including positions, velocities and dynamic parameters (e.g. the solar radiation pressure parameters (SRP)) of the satellite. $F(x, t)$ is the modeling equation of the complete set of forces acting on an orbiting satellite (G. Beutler,2004; McCarthy and Petit (eds.),2004). With proper integration method such as Adams-Cowell numerical integration, dynamic integrated orbits $x^{*}$ can be computed based on $x_{0}$. 
In equation (1), we can define $\delta=x-x^{*}$. Based on the Taylor expansion, we get

$$
\dot{\delta}=\left.\frac{\partial F(x, t)}{\partial x}\right|_{*} \delta
$$

The solution of (2) can be expressed as,

$$
\delta=\Psi\left(t, t_{0}\right) \delta_{0}
$$

Where, $\delta_{0}=x_{0}-x^{*}$ is the orbit corrections at initial epoch $t_{0}$. Substitute (3) into (2), we have the following equation,

$$
\left\{\begin{array}{l}
\dot{\Psi}\left(t, t_{0}\right)=\frac{\partial F}{\partial x} \Psi\left(t, t_{0}\right) \\
\Psi\left(t_{0}, t_{0}\right)=I
\end{array}\right.
$$

Where, $I$ is the unit matrix, $\Psi\left(t, t_{0}\right)$ is called transition matrix. It can be expressed in detail as,

$$
\Psi\left(t, t_{0}\right)=\left(\begin{array}{lll}
\frac{\partial r}{\partial r_{0}} & \frac{\partial r}{\partial \dot{r}_{0}} & \frac{\partial r}{\partial p} \\
\frac{\partial \dot{r}}{\partial r_{0}} & \frac{\partial \dot{r}}{\partial \dot{r}_{0}} & \frac{\partial \dot{r}}{\partial p} \\
\frac{\partial p}{\partial r_{0}} & \frac{\partial p}{\partial \dot{r}_{0}} & \frac{\partial p}{\partial p}
\end{array}\right)
$$

From numeric integration we can get transition matrix as well as integrated orbits $x^{*}$.

\section{REDUCED-DYNAMIC POD BASED ON HELMERT TRANSFORMATION}

Helmert transformation is mostly used to express differences between reference frames (C. Boucher, et al, 2004). It considers the origin motions and frame rotations. It is also used by the International GNSS Service (IGS, http://igscb.jpl.nasa.gov/) community to analyze the systematic differences between Analysis Centers (ACs) and to combine products from different ACs to get the final IGS products (G. Beutler, 1995). Considering the systematic differences, we can build up Helmert transformation between dynamic integrated orbits and kinematic orbits. At epoch $t_{i}$ it can be expressed as,

$$
\left(\begin{array}{c}
X_{K}^{i} \\
Y_{K}^{i} \\
Z_{K}^{i}
\end{array}\right)=\left(\begin{array}{c}
\Delta X \\
\Delta Y \\
\Delta Z
\end{array}\right)+\left(1+K^{\prime}\right) R_{1}\left(\alpha^{\prime}\right) R_{2}\left(\beta^{\prime}\right) R_{3}\left(\gamma^{\prime}\right)\left(\begin{array}{c}
X_{T}^{i} \\
Y_{T}^{i} \\
Z_{T}^{i}
\end{array}\right)
$$

Where, $\left(X_{K}^{i}, Y_{K}^{i}, Z_{K}^{i}\right)$ are kinematic orbits. $\left(X_{T}^{i}, Y_{T}^{i}, Z_{T}^{i}\right)$ are dynamically integrated orbits expressed in Earth-fixed reference frame, which can be obtained using the following transformation (McCarthy and Petit (eds.),2004), 


$$
\left(\begin{array}{c}
X_{T}^{i} \\
Y_{T}^{i} \\
Z_{T}^{i}
\end{array}\right)=Q\left(t_{i}\right) R\left(t_{i}\right) W\left(t_{i}\right)\left(\begin{array}{c}
X_{I}^{i} \\
Y_{I}^{i} \\
Z_{I}^{i}
\end{array}\right)
$$

Where, $\left(X_{I}^{i}, Y_{I}^{i}, Z_{I}^{i}\right)$ are integrated orbits expressed in inertial reference frame, $Q\left(t_{i}\right), R\left(t_{i}\right), W\left(t_{i}\right)$ are the matrices for precession-nutation, Earth rotation and pole wobble, respectively.

We can rewrite the model as,

$$
r_{K}^{i}=T+(1+K) R 1 \cdot R 2 \cdot r_{I}^{i}
$$

Where, $r_{K}^{i}, r_{I}^{i}$ are the denotation of kinematic orbit (in Earth-fixed frame), dynamic orbit (in inertial frame), $T, K$ represent the translation and the scale parameters of the Helmert transformation, and $R 1, R 2$ are rotation matrices in equation (6) and (7). In $R 2$, parameters contained are: Earth pole $x_{p}, y_{p}$ and the rates $\dot{x}_{p}, \dot{y}_{p}$, time parameter $U T 1-U T C(d U T 1)$ and the rate $d \dot{U} T 1$.

The magnitude of $x_{p}, y_{p}$ is less than $1 "$ and the magnitude of $d U T 1$ is less than 1 second (15" in angle), Magnitude of $\dot{x}_{p}, \dot{y}_{p}$ and $d \dot{U} T 1$ are even smaller. Ignoring the effects of $\dot{x}_{p}, \dot{y}_{p}$ and $d \dot{U} T 1$, equation (8) can be rewritten as,

$$
\left(\begin{array}{c}
X_{K}^{i} \\
Y_{K}^{i} \\
Z_{K}^{i}
\end{array}\right)=\left(\begin{array}{c}
\Delta X \\
\Delta Y \\
\Delta Z
\end{array}\right)+(1+K) R_{1}(\alpha) R_{2}(\beta) R_{3}(\gamma) R_{e t c} \cdot\left(\begin{array}{c}
X_{I}^{i} \\
Y_{I}^{i} \\
Z_{I}^{i}
\end{array}\right)
$$

Where, $R_{e t c}$ is the residual matrix comparing equation (9) to equation (8) and,

$$
\alpha=\alpha^{\prime}+y_{p}, \quad \beta=\beta^{\prime}+x_{p}, \quad \gamma=\gamma^{\prime}+d U T 1
$$

Rewrite equation (10) as following,

$$
r_{K}^{i}=T+(1+K) R \cdot R_{e t c} \cdot r_{I}^{i}
$$

Where, $T, K, R$ represent Helmert transformation parameters $(\Delta X, \Delta Y, \Delta Z, K, \alpha, \beta, \gamma)$.

The linearization of equation (11) reads as, 


$$
r_{K}^{i}=r_{K, 0}^{i}+\frac{\partial r_{K}^{i}}{\partial v} d v
$$

Parameters to be estimated are expressed in equation (13), where $\mathrm{dHel}$ are corrections of Helmert transformation parameters, $d r_{I}^{i}$ are corrections of integrated orbits at current epoch.

$$
d v=\left(d H e l, d r_{I}^{i}\right)^{T}
$$

Considering equation (3) and (4), we can transform the parameter $d r_{I}^{i}$ to initial orbit corrections $d r_{0}$ using equation (14).

$$
d r_{I}^{i}=\Psi\left(t_{i}, t_{0}\right) d r_{0}
$$

Therefore the final parameters can be expressed as:

$$
d x=\left(d H e l, d r_{0}\right)^{T}
$$

Design matrix is:

$$
A_{i}=\frac{\partial r_{K}^{i}}{\partial x}=\left(\frac{\partial r_{K}^{i}}{\partial H e l}, \frac{\partial r_{K}^{i}}{\partial r_{I}^{i}} \cdot \Psi\left(t_{i}, t_{0}\right)\right)
$$

Equation (12) can be formed at each epoch $t_{i}$. The solution of these equations may be obtained by classical Least Square Estimation (LSE).

\section{DATA PROCESSING}

To implement the reduced-dynamic method introduced above, a set of programs are developed. Data processing procedure contains the following two parts:

- Kinematic orbit determination and,

- Reduced-dynamic orbit determination

The procedure runs in iterations. It starts with kinematic orbit determination. The basis algorithm in kinematic POD is the combination of the orbits calculated from code observations and the orbit differences based on phase-differences between consequent epochs (H. Bock, 2003). Afterwards, reduced-dynamic orbit determination is performed using the proposed model. The reduced-dynamic orbits can be later used in data pre-screening of kinematic POD during iterations. The final reduced-dynamic orbits are our best results. Applying this procedure, POD of CHAMP and GRACE was performed.

\subsection{CHAMP POD}

Onboard GPS observations and accelerometer measurements in Day of Year (DoY) 191, 2004, were used. Kinematic orbits were first derived with an RMS of $0.38 \mathrm{~m}$ compared to GFZ Post-processed Science Orbit (PSO, http://isdc.gfz-potsdam.de/). The initial orbits (positions, 
velocities and 9 SRP parameters) of reduced-dynamic POD are derived from kinematic orbits by interpolation. Dynamic models and parameter settings are listed in table 1.

Table 1. Dynamic models of CHAMP

\begin{tabular}{|l|l|}
\hline Gravity model & EIGEN-CHAMP03S (120*120) \\
\hline Tide & Solid Earth Tide,Ocean Tide CSR 3.0 \\
\hline N-body & JPL ephemeris DE405 \\
\hline Accelerometer data & ACC File(with official bias and scale parameter) \\
\hline
\end{tabular}

Additionally, 9 empirical parameters ( 3 constant and 6 periodic terms) are set up in the inertial system for each revolution of the satellite's trajectory, which is similar with the empirical parameters described in (H. Bock, 2003). All together 144 empirical parameters are set up for this day. The performance of different parameter sets were studied first. Different sets of Helmert parameters are listed in table 2, where the first case (Ex.1) is the conventional orbit fitting (e.g. D. Svehla, 2003; H. Bock, 2003). Figure 1 shows the RMS of the final reduced-dynamic orbits compared to GFZ PSO.

Table 2. Parameter settings, with $\times$ indicates that the parameter is set up

\begin{tabular}{|c|c|c|c|c|c|c|c|c|}
\hline & Ex. 1 & Ex. 2 & Ex. 3 & Ex. 4 & Ex. 5 & Ex. 6 & Ex. 7 & Ex. 8 \\
\hline$T$ & & $\times$ & & $\times$ & & $\times$ & & $\times$ \\
\hline$K$ & & & $\times$ & $\times$ & & & $\times$ & $\times$ \\
\hline$R$ & & & & & $\times$ & $\times$ & $\times$ & $\times$ \\
\hline
\end{tabular}

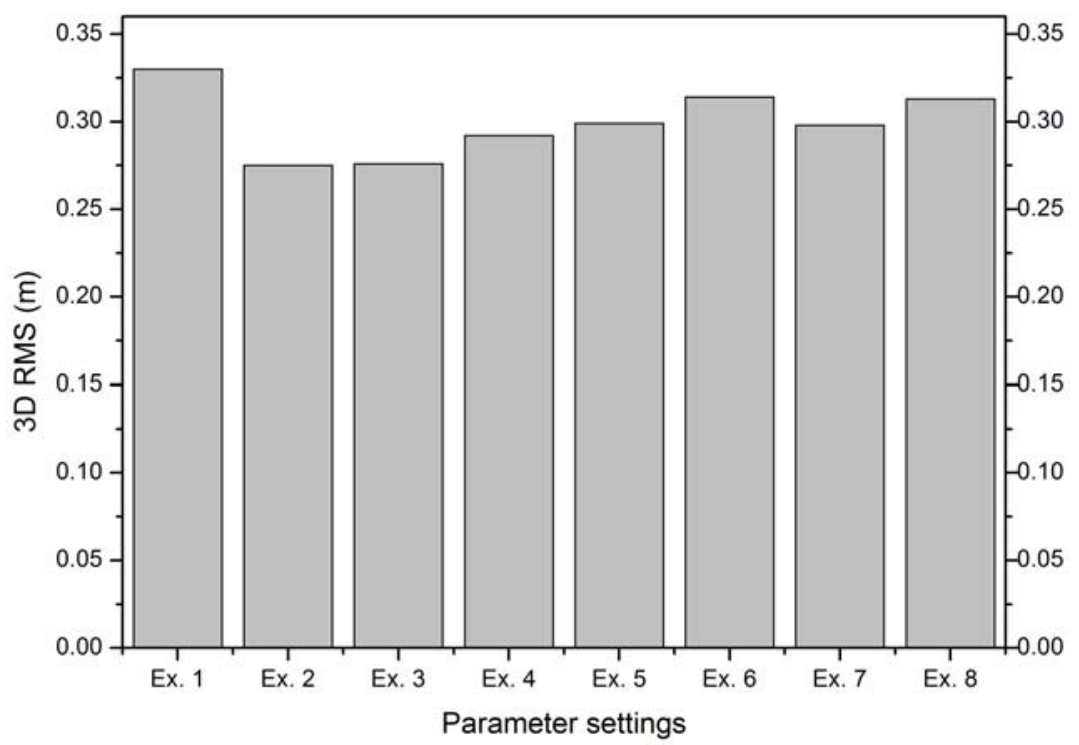

Fig. 1. RMS of orbit differences between CHAMP reduced-dynamic orbits and GFZ PSO, under different parameter settings

The RMS of reduced-dynamic orbits in case 1 , which is $0.33 \mathrm{~m}$, is reduced compared to kinematic orbits. Using our new model, we obtain better orbits with the parameter settings from case 2 to case 8 , where in case 2 and case 3 the orbit precision is the best. The RMS is almost the same in case 5 and case 7 , also in case 6 and case 8, which means that the scale parameter may be not sensitive in our model when rotation parameters are set up. But scale 
parameter does contribute to our model, this can be clearly seen when we compare the results in case 1 to that in case 3 (where scale parameter is estimated).

The Helmert parameters estimated from different cases show that:

(1) the translation parameters have differences within $2 \mathrm{~cm}$,

(2) the scale parameter has small differences in different solutions (less than 0.5 $\mathrm{ppb}$ ), which is due to the low correlation between scale parameter and other parameters, and

(3) the rotation parameters are almost the same in case 5 and case 6 and also similar in case 7 and case 8 . This may be due to the fact that the correlation of translation parameter and rotation parameters is low.

Table 3 shows the estimated Helmert parameters in case 8. As can be seen that rotation and scale parameters are remarkable, which reflects the systematic differences between our kinematic orbits and reduced-dynamic orbits.

Table 3. Estimated parameters, X,Y,Z indicates different axis

\begin{tabular}{|c|c|c|c|}
\hline & $\mathrm{X}$ & $\mathrm{Y}$ & $\mathrm{Z}$ \\
\hline$T(\mathrm{~cm})$ & $-0.21 \pm 0.08$ & $0.47 \pm 0.06$ & $1.32 \pm 0.06$ \\
\hline$R(\mathrm{mas})$ & $1.86 \pm 0.07$ & $3.87 \pm 0.14$ & $1.12 \pm 0.08$ \\
\hline$K(\mathrm{ppb})$ & $-5.52 \pm 0.28$ & \multicolumn{3}{|l|}{} \\
\hline
\end{tabular}

Figure 2 shows the orbit differences between our final reduced-dynamic orbits and PSO in case 2. The differences are expressed in the satellite spacecraft system (RSW system). The differences show (especially in along track) periodicities. The RMS is $0.27 \mathrm{~m}$, which is reduced by $29 \%$ compared to kinematic orbits. The RMS is $(0.13,0.18,0.14) \mathrm{m}$ in along track, cross track and radial direction, respectively. The mean of orbit differences is $(-0.2,0.4,-0.5)$ $\mathrm{cm}$.

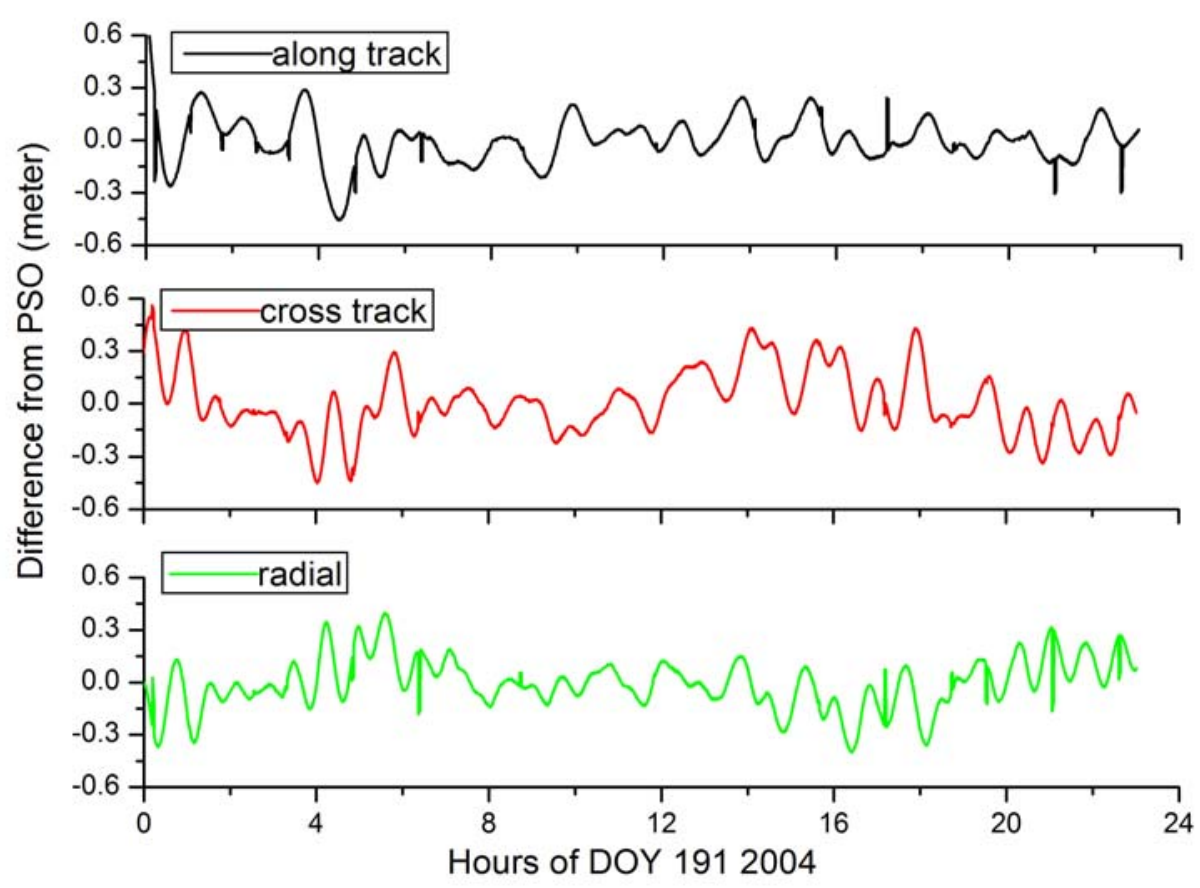

Fig. 2. Difference between CHAMP reduced-dynamic orbits and GFZ PSO, 3DRMS=0.27 m 


\subsection{GRACE POD}

GRACE-A onboard GPS observations and accelerometer measurements in DoY 094, 2003, were used. Kinematic orbits were first derived with an RMS of $0.39 \mathrm{~m}$ compared to GNV1B (Kelley Case et al, 2004) orbits of JPL. Nine empirical parameters are set up in the inertial system for each revolution of the satellite's trajectory, gravity model used is EIGEN-GRACE02S (http://icgem.gfz-potsdam.de/ICGEM/), and other dynamic parameters are the same as in table 1.

Under the parameter setting case 2 (Ex.2) in table 2, reduced-dynamic POD for GRACE was carried out. Figure 3 shows the difference between reduced-dynamic orbits and GNV1B orbits. Except the periodicities, the orbit differences also show dithering effects. This may caused by the high rate accelerometer measurements (we use 1-Hz measurements). The RMS is $0.22 \mathrm{~m}$, which is reduced by $44 \%$ compared to kinematic orbits. RMS is $(0.11,0.12,0.14)$ $\mathrm{m}$ in along track, cross track and radial direction, respectively. The mean of orbit differences is $(1.1,-1.2,-1.7) \mathrm{cm}$.

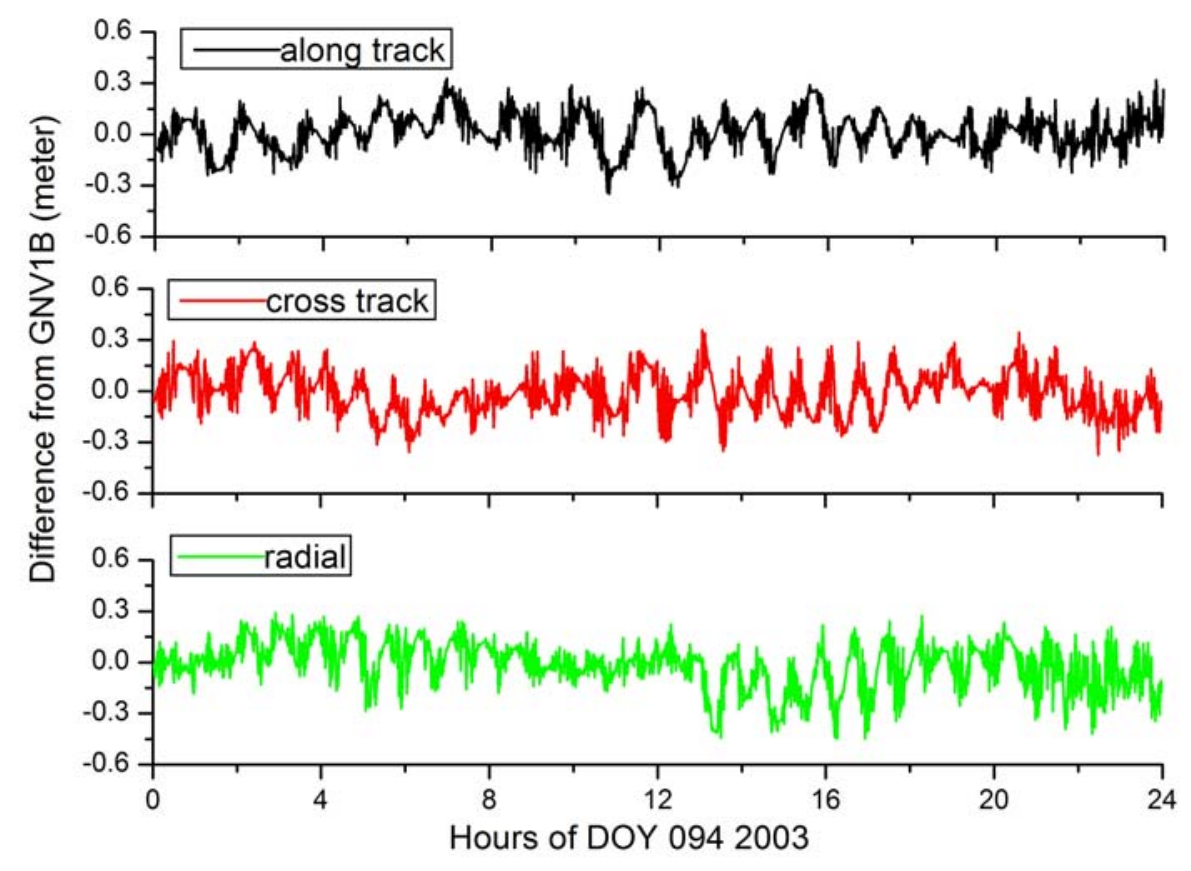

Fig. 3. Difference between GRACE reduced-dynamic orbits and GNV1B orbits, $3 \mathrm{DRMS}=0.22 \mathrm{~m}$

\subsection{FURTHER VALIDATION}

As a further investigation of our model, data of CHAMP and GRACE-A from DoY 124 to DoY 137, 2003 were processed. Figure 4 and 5 show the RMS statistic of the reduced-dynamic orbits compared to the reference orbits. 


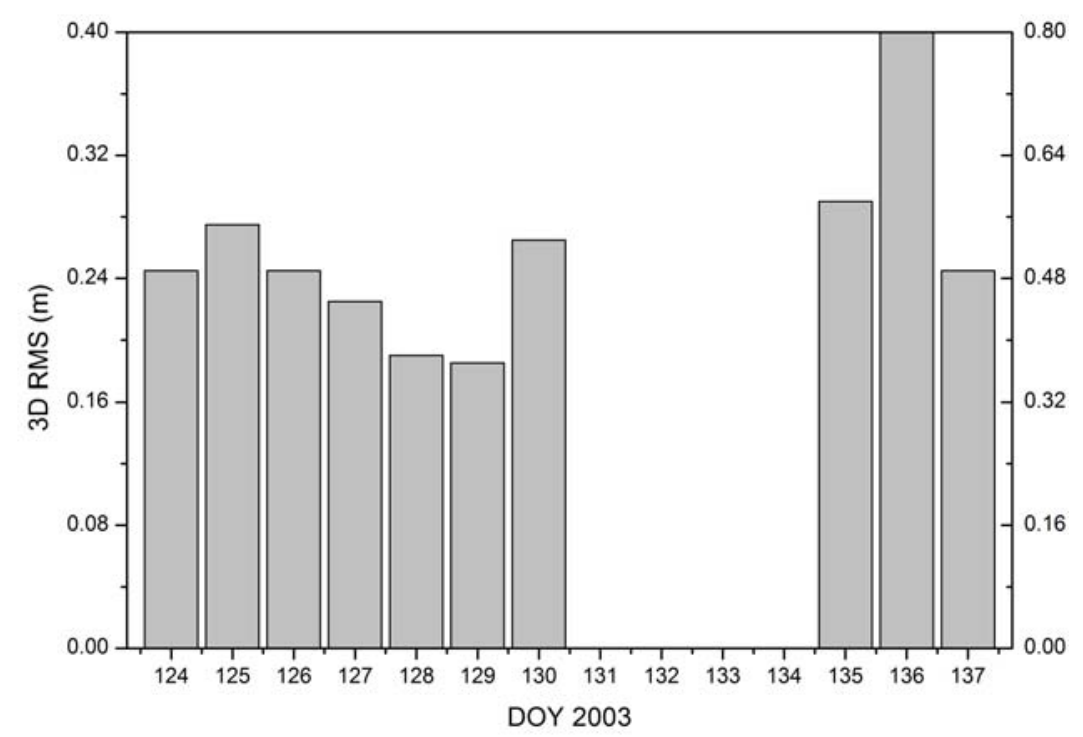

Fig. 4. RMS of the difference between CHAMP reduced-dynamic orbits and PSO

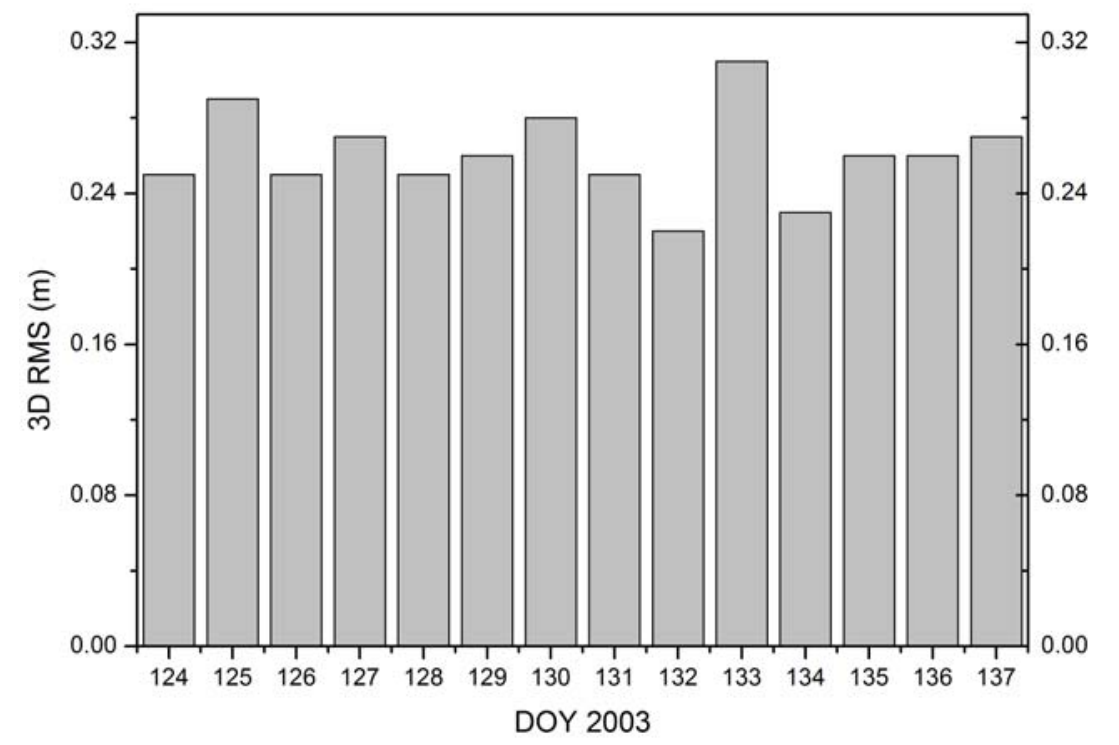

Fig. 5. RMS of the difference between GRACE reduced-dynamic orbits and GNV1B

The comparison for CHAMP during DoY 131-134 is not performed, because the GFZ PSOs are missing in these days.

As is shown in figure 4 and figure 5, the mean 3D RMS of both CHAMP and GRACE orbits is $0.26 \mathrm{~m}$.

\section{CONCLUSIONS}

As POD performed for CHAMP and GRACE shows, the proposed model reduces the errors of kinematic orbits in reduced-dynamic POD and removes the systematic difference between kinematic orbits and reduced-dynamic orbits. Therefore the orbit precision improves by around $30 \%-45 \%$.

The investigation of different parameter settings of our model shows that the introduction of Helmert transformation parameters improves orbit precision, and orbits achieve the highest precision under the parameter setting with only transformation parameters being estimated. 
Tests of our model also show that the estimated Helmert parameters have only small differences under different parameter settings.

The reduced-dynamic orbits derived from our research have obvious periodicities, which follows, in general, the revolution periods of the satellites. This may be due to the errors in accelerometer measurements, which are corrected only using the official calibration parameters in our research. Further step to add calibration parameters of accelerometer measurements in our approach needs to be investigated.

Acknowledgement: The authors thank Dr. Maorong Ge from GeoForschungsZentrum Potsdam for giving many suggestions in this research.

\section{REFERENCES}

[1] Christoph Reigber, Hermann Luehr and Peter Schwintzer (eds.), First CHAMP Mission Results for Gravity, Magnetic and Atmospheric Studies. Springer-Verlag Berlin Heidelberg 2003

[2] C. Boucher, Z. Altamimi, P. Sillard, and M. Feissel-Vernier, The ITRF2000 (IERS Technical Note No. 31). Frankfurt am Main: Verlag des Bundesamts für Kartographie und Geodäsie ,2004.

[3] Chen, Jun-ping, Wang, Jie-xian. Solar Radiation Pressure Models for the GPS Satellites. Acta Astronomica Sinica, 1998(3), Vol 47, pp. 310-319, 2006.

[4] D. D. McCarthy and Gérard Petit (eds.), IERS Conventions (2003) (IERS Technical Note No. 32).Frankfurt am Main: Verlag des Bundesamts für Kartographie und Geodäsie, 2004

[5] D. Svehla, M. Rothacher, KINEMATIC AND REDUCED-DYNAMIC PRECISE ORBIT DETERMINATION OF CHAMP SATELLITE OVER ONE YEAR USING SPACEBORNE GPS PHASE ZERO-DIFFERENCES ONLY. Geophysical Research Vol.5 12129, 2003

[6] Gerhard Beutler, Methods of Celestial Mechanics Volume I: Physical, Mathematical, and Numerical Principles. Springer-Verlag Berlin Heidelberg, 2004

[7] Gerhard Beutler, Methods of Celestial Mechanics Volume II: Application to Planetary System, Geodynamics and Satellite Geodesy. Springer-Verlag Berlin Heidelberg, 2004

[8] G. Beutler, J. Kouba and T. Springer, Combining the Orbits of IGS Processing Centers. In: Bulletin Géodésique Vol. 69, pp. 200-222 No. 4, 1995

[9] H. Bock. Efficient Methods for Determining Precise Orbits of Low Earth Orbiters Using the Global Positioning System. Phd thesis, Astronomical Institute University of Berne, Switzerland, 2003

[10] Junping Chen. On precise orbit determination of low earth orbiters. Phd thesis, Shanghai,Tongji University, 2007.

[11] Kelley Case,Gerhard L. H. Kruizinga,Sien-Chong Wu. GRACE Level 1B Data Product User Handbook. JPL,2004 
[12] S.Zhu, Ch. Reigber, R. Koenig. Integrated adjustment of CHAMP, GRACE, and GPS data. Journal of Geodesy (2004) 78: 103-108,2004

Received: 2008-01-23,

Reviewed: 2008-04-28,

Accepted: 2008-06-04. 